\title{
Relación del Color y del Estado de Madurez con las Propiedades Fisicoquímicas de Frutas Tropicales
}

\author{
Ramiro Torres*, Everaldo J. Montes, Omar A. Pérez y Ricardo D. Andrade \\ Universidad de Córdoba, Departamento de Ingeniería de Alimentos, Facultad de Ingenierías, \\ Grupo de Investigación GIPPAL, Km 12 Vía Cereté-Ciénaga de Oro, Colombia \\ (e-mail:rtorres@correo.unicordoba.edu.co) \\ * Autor a quien debe ser dirigida la correspondencia.
}

Recibido Nov. 21, 2012; Aceptado Dic. 28, 2012; Versión final recibida Ene. 02, 2013

\begin{abstract}
Resumen
Se ha determinado la relación del color y estado de madurez con las propiedades fisicoquímicas de frutas tropicales (mango puerco y corazón, maracuyá, papaya y plátano). El color fue medido a partir de un sistema de adquisición de imágenes, expresado en el espacio cromático CIELAB. El pH e índice de madurez presentaron buen ajuste a modelos lineales y polinómicos con los parámetros de color. $\mathrm{El} \mathrm{pH}$ aumentó levemente con el incremento del estado de madurez, mientras que la acidez titulable disminuye en el mango, maracuyá y papaya, debido al efecto amortiguador del ácido cítrico. En el plátano el pH disminuye con el aumento del estado de madurez y la acidez titulable aumenta. Esto ocurre como resultado de la degradación de almidones en azúcares reductores y su conversión en ácido pirúvico.
\end{abstract}

Palabras clave: frutas tropicales, sistema de adquisición de imágenes, color, índice de madurez, CIELAB

\section{Relation of Color and Maturity Stage with Physicochemical Properties of Tropical Fruits}

\begin{abstract}
In this study, the relationships of color and maturity stage with the physicochemical properties of tropical fruits (puerco and corazón varieties of mango, passion fruit, papaya and plantain) were investigated. The color of tropical fruits was measured using image acquisition system, expressed in CIELAB color space. The $\mathrm{pH}$ and the maturity index showed good fitting with the color parameters using linear and polynomial models. The $\mathrm{pH}$ increased slightly with increasing maturity stage, while titratable acidity decreases in mango, passion fruit and papaya, due to cushioning effect of citric acid. In the plantain, the $\mathrm{pH}$ decreases with increasing maturity index. However, titratable acidity increases with increasing maturity stage. This occurs as result of the degradation of starch in sugars and their conversion into pyruvic acid.
\end{abstract}

Keywords: tropical fruits, image acquisition system, color, maturity index, CIELAB 


\section{INTRODUCCIÓN}

La producción mundial de frutas tropicales en el año 2010 fue de aproximadamente 62 millones de toneladas, y se estima que para el 2014 la producción ascenderá a 82,1 millones de toneladas. La variedad dominante fue el mango con el $36 \%$ de la producción mundial de frutas tropicales, seguido de la piña, papaya, aguacate, guayaba y maracuyá. Los volúmenes del comercio indican que América Latina y el Caribe realizó el $61 \%$ de las exportaciones mundiales de las principales frutas tropicales frescas, mientras que el Lejano Oriente y África le correspondió el 22 y 10\%, respectivamente (FAO, 2012; Torres et al., 2012).

Los principales parámetros de calidad de las frutas son el peso seco, sólidos solubles, acidez titulable, pH, color y dureza. Generalmente, frutas como el mango, papaya, maracuyá y plátano, se consumen con su máxima calidad organoléptica, que se presenta cuando la fruta ha alcanzado por completo el color amarillo o naranja(característico de madurez), pero antes del ablandamiento excesivo. Por tanto, el color en estas frutas es la característica externa más importante en la determinación del punto de maduración y de la vida poscosecha y un factor determinante en la decisión de compra por parte de los consumidores. El color amarillo es el resultado de la degradación de la clorofila, así como de la síntesis de cromoplastos (Bruhn, 2007; Fraser et al., 1994).

Un gran número de procesos fisicoquímicos se llevan a cabo en las frutas durante el almacenamiento, período en el cual la calidad de la mayoría de frutas y hortalizas se ve severamente afectada por las pérdidas de agua, que dependen de la temperatura y de la humedad relativa. Sin embargo, el efecto de la temperatura de almacenamiento sobre la calidad y la cantidad de cambios fisicoquímicos en frutos es altamente dependiente del cultivar, el tiempo de exposición y las condiciones de cosecha (Casierra y Aguilar, 2008; Moccia et al., 2006).

Las propiedades fisicoquímicas de las frutas se realizan con equipos y procedimientos especializados y personal entrenado, lo que genera altos costo de análisis. Sin embargo, estas se pueden relacionar con atributos o propiedades fáciles de medir y que no impliquen análisis destructivos, como por ejemplo el color (Segnini et al., 1999; Abdullah et al., 2001). El color se puede analizar rápidamente por un sistema de adquisición de imágenes (Mendoza et al., 2006), también conocidas como sistemas de visión computacional (SVC). El SVC ha sido utilizado para identificar, clasificar, evaluar y detectar defectos en frutas, verduras, carnes, productos de panadería, y productos preparados, como chips de papas fritas (Pedreschi et al., 2004).Aunque para la medida del color, actualmente están disponibles colorímetros comerciales en espacio LAB, estos tienen como desventaja con respecto al SVC, que la superficie a medir debe ser uniforme y pequeña (aproximadamente $2 \mathrm{~cm}^{2}$ ) lo que hace que las medidas sean poco representativas en materiales heterogéneos tales como la mayoría de las frutas (Leon et al., 2006).

El objetivo de esta investigación fue determinar el color por la técnica de visión computacional, y correlacionarlo con las características fisicoquímicas del mango (variedades puerco y corazón), maracuyá amarilla, papaya Hawaiana y plátano; además de determinar la influencia del estado de madurez sobre las características fisicoquímicas de estas frutas.

\section{MATERIALES Y MÉTODOS}

\section{Materia Prima}

Se utilizaron frutos de mango (Mangifera indica L) en las variedades puerco y corazón, maracuyá amarilla (Passifloraedulisvar. FlavicarpaDegener), papaya Hawaiana (Carica papaya L.) y plátano (Mussa Cavendish), provenientes de Montería, Córdoba (Colombia), en diferentes estados de madurez, los cuales fueron identificados visualmente por el color de la cáscara mediante la escala de maduración para mango (Peña, 2008), plátano (Dadzie y Orchard, 1997), maracuyá y papaya (Santamaría et al., 2009).

\section{Parámetros de Color}

La determinación del color se realizó por adquisición de imágenes, con un sistema similar al reportado por León et al. (2006), utilizando lámparas fluorescentes (60 cm longitud) con una temperatura de color de 6500 K (Philips, luz del día natural, 18W), una cámara digital de color (CDC) marca Canon PowerShot SX1IS (Canon ${ }^{\circledR}$, Tokio, Japón) con 10.0 Mega pixeles de resolución. Las imágenes fueron capturadas empleando máxima resolución (pixeles $4000 \times 3000$ ). La CDC se configuró según lo propuesto por León et al. (2006), utilizando una carta de color Colorchecker $24 \circledast$. 
Para la cuantificación del color en el espacio CIELAB (Commissionlnternationale de l'Eclairage $L^{*}, a^{*}$ y b*), se utilizó el software Adobe Photoshop CS5® (Adobe Systems inc., 2012). Se seleccionó la imagen de la fruta capturada en la foto, usando la opción "herramienta varita mágica" que permite filtrar el fondo y en la ventana "histograma" del software se obtienen el gráfico de la distribución acumulada, media, desviación estándar para cada uno de los parámetros ( $L$, a y b) y número de pixeles. Los valores de los parámetros $L$, a y $b$ se convirtieron al espacio de color de CIELAB utilizando transformaciones lineales propuestas por Yam y Papadakis(2004). Con los valores L* (Luminosidad), $a^{*}$ (componente rojo-verde) y b* (componente amarillo - celeste) de cada muestra se calcularon los valores tono ( $\left.{ }^{\circ} \mathrm{HUE}\right)$ y croma $\left(\mathrm{C}^{*}\right)$.

\section{Estados de madurez}

El estado de madurez (EM) se determinó de acuerdo al color de la fruta expresada en los parámetros CIELAB, siguiendo la metodología propuesta por Torres et al. (2012).

\section{Propiedades fisicoquímicas}

La concentración de sólidos solubles se determinó por el método AOAC 932.12 (AOAC, 1990), utilizando un refractómetro digital (Mettler Toledo-30PX $®$ ); la acidez titulable por el método AOAC 942.15 (AOAC, 1990), el pH según método AOAC 981.12/90 (AOAC, 1990) con potenciómetro digital (Mettler Toledo S40 $\left.{ }^{\circledR}\right)$. El cálculo del índice de madurez (IM) se determinó como la relación entre sólidos solubles y acidez titulable.

\section{Análisis estadístico}

El experimento para la determinación del pH e índice de madurez, en la papaya Hawaiana y el plátano, fue conducido bajo un diseño completamente aleatorizado (DCA) unifactorial, con el factor EM en 7 niveles (1, $2,3,4,5,6$ y 7 ), con 3 repeticiones (21 unidades experimentales); de igual manera en la maracuyá variedad amarilla con el mismo factor, pero en 6 niveles. Para el mango el experimento se realizó bajo un diseño en bloques completamente aleatorizado (DBCA), unifactorial para el estado de madurez en 6 niveles (1, 2, 3, 4, 5 y 6), bloqueando el factor variedad (puerco y corazón), con 3 repeticiones para un total de 36 unidades experimentales. Para determinar la influencia del EM sobre el IM y $\mathrm{pH}$, se realizó un análisis de varianza (ANOVA) y test de Tukey a un nivel de significancia del 5\%.La variación del pH e IM con respecto los parámetros de color se ajustaron a ecuaciones por análisis de regresión lineal. Para determinar la validez de las ecuaciones se utilizóel coeficiente determinación $\left(R^{2}\right)$ y el análisis de los residuos.

\section{RESULTADOS Y DISCUSIONES}

\section{Correlación entre color y propiedades fisicoquímicas}

En la tabla 1 se presentan las ecuaciones de ajuste para predecir el pH en función de los parámetros de color en coordenadas rectangulares y cilíndricas para el mango variedades puerco y corazón, maracuyá amarilla, papaya hawaiana y plátano. El ajuste de datos experimentales del color en el espacio de color CIELAB por regresión lineal múltiple, en coordenadas rectangulares $\left(L^{*}, a^{*}\right.$ y $\left.b^{*}\right)$ muestra que la ecuación de ajuste del $\mathrm{pH}$ se ve afectado para el mango variedad puerco por $a^{*}(p=0,0015)$ y $b^{*}(p=0,002)$, mango variedad corazón por $a^{*}(p=0,0010)$ y $b^{*}(p=0,0014)$, maracuyá por $a^{*}(p=0,0003)$, papaya hawaiana por $a^{\star}$ $(p=0,0002)$ y plátano por $a^{*}(p=0.0001)$. En coordenadas cilíndricas $\left(L^{*},{ }^{\circ} \mathrm{HUE}, \mathrm{C}^{\star}\right)$ muestra que el croma $\left(C^{\star}\right)$ contribuye significativamente $(5 \%$ de significancia) al modelo del pH para el mango en las variedades puerco $(p=0,0004)$ y corazón $(p=0,0001)$; mientras que el tono $\left({ }^{\circ} \mathrm{HUE}\right)$ contribuye al modelo del pH para todas las frutas evaluadas.

Tabla 1: Ecuación de ajuste del pH con los parámetros de color

\begin{tabular}{|c|c|c|}
\hline Fruta & Ecuación & $\mathrm{R}^{2}$ \\
\hline $\begin{array}{c}\text { Mango variedad } \\
\text { puerco }\end{array}$ & $\mathrm{pH}=3,03+0,01 \mathrm{a}^{*}+0,01 \mathrm{~b}^{*}$ & 0,993 \\
\hline $\begin{array}{c}\text { Mango variedad } \\
\text { corazón }\end{array}$ & $\mathrm{pH}=4,06-0,01$ HUE $+0,01 \mathrm{C}^{*}$ & 0,996 \\
\hline Maracuyá & $\mathrm{pH}=4,31-0,0101 \mathrm{a}^{*}+0,01 \mathrm{~b}^{*}$ & 0,979 \\
variedad amarilla & $\mathrm{pH}=2,87-01 \mathrm{C}^{*}$ & 0,976 \\
\hline Papaya variedad & $\mathrm{pH}=1,63+0,01$ oHUE & 0,869 \\
Hawaiana & $\mathrm{pH}=5,90+0,01 \mathrm{a}^{*}$ & 0,875 \\
\hline Plátano & $\mathrm{pH}=6,63-0,01$ OHUE & 0,873 \\
& $\mathrm{pH}=4,28-0,06 \mathrm{a}^{*}$ & 0,871 \\
\hline & $\mathrm{pH}=-0,05+0,05$ HUE & 0,898 \\
\hline
\end{tabular}


El ajuste de datos experimentales del color en el espacio de color CIELAB por regresión lineal múltiple, en coordenadas rectangulares $\left(L^{*}, a^{*}\right.$ y $\left.b^{*}\right)$ muestra que la ecuación de ajuste del índice de madurez (IM) el parámetro $a^{*}$ contribuye significativamente para el mango en la variedad puerco, corazón, maracuyá, papaya, y plátano; $b^{*}$ contribuye solamente en el plátano, mientras que el parámetro $L^{*}$ no contribuye significativamente para las frutas estudiadas. En coordenadas cilíndricas ( $\left.L^{*},{ }^{\circ} \mathrm{HUE}, C^{*}\right)$ muestra que la luminosidad $\left(L^{*}\right)$ no contribuye significativamente ( $5 \%$ de significancia) al modelo de IM para el mango y papaya; $C^{*}$ contribuye significativamente a la ecuación de ajuste para la papaya y ${ }^{\circ} \mathrm{HUE}$ contribuye para la predicción del índice de madurez. En la tabla 2 se presentan las ecuaciones de ajuste para predecir el índice de madurez con los parámetros de color, mostrando estas ecuaciones buen ajuste $\left(R^{2}>0,81\right)$.

Tabla 2:Ecuación de ajuste del Índice de Madurez (IM)con los parámetros de color.

\begin{tabular}{|c|c|c|}
\hline Fruta & Ecuación & $\mathrm{R}^{2}$ \\
\hline $\begin{array}{l}\text { Mango variedad } \\
\text { puerco }\end{array}$ & $\begin{array}{c}I M=43,88+1,68 a^{*} \\
I M=178,17-1,49^{\circ} \mathrm{HUE}\end{array}$ & $\begin{array}{l}0,961 \\
0,950\end{array}$ \\
\hline $\begin{array}{l}\text { Mango variedad } \\
\text { corazón }\end{array}$ & $\begin{array}{c}I M=51,43+1,82 a^{*} \\
I M=198,75-1,69^{\circ} \mathrm{HUE}\end{array}$ & $\begin{array}{l}0,911 \\
0,928\end{array}$ \\
\hline $\begin{array}{l}\text { Maracuyá variedad } \\
\text { amarilla }\end{array}$ & $\begin{array}{c}I M=4,79-0,07 L^{*}+0,82 a^{*}+0,04 b^{*} \\
I M=14,20-0,08 L^{*}-0,09^{\circ} H U E\end{array}$ & $\begin{array}{l}0,951 \\
0,948\end{array}$ \\
\hline $\begin{array}{l}\text { Papaya variedad } \\
\text { Hawaiana }\end{array}$ & $\begin{array}{c}I M=108,55+4,60 a^{*}+0,07\left(a^{*}\right)^{2} \\
I M=1517,83-3,35^{\circ} H U E-2,07 C^{*}\end{array}$ & $\begin{array}{l}0,984 \\
0,956\end{array}$ \\
\hline Plátano & $\begin{array}{c}I M=54,81-3,02 L^{*}+2,02 a^{*}+4,06 b^{*} \\
I M=-11447,28+30,39^{\circ} H U E-0,15\left({ }^{\circ} H U E\right)^{2}\end{array}$ & $\begin{array}{l}0,819 \\
0,940\end{array}$ \\
\hline
\end{tabular}

Influencia del estado de madurez sobre las propiedades fisicoquímicas

El ANOVA muestra que el pH se ve influenciado (significancia del 5\%) por el estado de madurez para todas las frutas. En la tabla 3 se observa que para el mango, maracuyá y papaya, un incremento en el estado de madurez ocasiona un aumento en el pH y una disminución de la acidez titulable, mientras que para el plátano un aumento en el EM produce una disminución del $\mathrm{pH}$ y un aumento de la acidez titulable. El poco aumento de $\mathrm{pH}$ (aproximadamente 15\%) con el EM en frutas como mango, maracuyá y papaya, se puede explicar por la presencia de un sistema de autorregulación del $\mathrm{pH}$, resultado del efecto amortiguador del ácido cítrico, como ha sido descrito para diversos frutos (Menéndez et al., 2006). Este ácido alifático tiende a convertirse en la sal correspondiente, dando como resultado el efecto amortiguador de la solución en la región de sus valores de pKa, disminuyendo la acidez (aproximadamente 50\%). La disminución del pH y aumento de la acidez titulable en el plátano, está relacionado con la degradación de almidón en azúcares reductores o su conversión en ácido pirúvico (Azcón-Bieto y Talón 2009) concordando con resultados obtenidos por Barrera et al. (2010), y Chang-Yuen y Sáenz (2005).

Para todas las frutas evaluadas, a medida que incrementa el estado de madurez se presenta un aumento en los sólidos solubles y el índice de madurez. Este comportamiento en los sólidos solubles, se explica por la hidrólisis de diversos polisacáridos estructurales tales como almidón, pectinas de la pared celular, hasta sus componentes monoméricos básicos, por lo cual se acumulan azúcares, principalmente glucosa, fructosa y sacarosa (Arrieta et al., 2006) que son los constituyentes principales de los sólidos solubles; estos resultados coinciden con lo encontrado por Sañudo et al. (2008). El mayor aumento en azúcares ocurre cuando el fruto empieza a tornarse amarillo acelerándose la síntesis de estos compuestos hasta alcanzar la coloración muy amarilla (Barrera et. al., 2010).

\section{CONCLUSIONES}

El pH se correlaciona con una ecuación lineal múltiple en $a^{*}$ y $b^{\star}$ (en ${ }^{\circ} H U E$ y $\left.C^{\star}\right)$ para el mango variedades puerco y corazón; con una ecuación lineal en $\mathrm{a}^{*}\left({ }^{\circ} \mathrm{HUE}\right)$ para la maracuyá amarilla, la papaya Hawaiana y el plátano. Mientras el índice de madurez se correlaciona con una ecuación lineal en $\mathrm{a}^{\star}$ ( $\left.{ }^{\circ} \mathrm{HUE}\right)$ para el mango variedades puerco y corazón; con una ecuación lineal múltiple en $L^{*}, a^{*}$ y $b^{*}$ para la maracuyá amarilla y el plátano, y con una ecuación polinómica de segundo grado en $a^{\star}$ para la papaya Hawaiana.

$\mathrm{El} \mathrm{pH}$ es influenciado por el aumento del EM, aunque presenta solo un aumento de aproximadamente de $15 \%$ para el mango, maracuyá y papaya, indicando la presencia de un sistema de autorregulación del pH. Mientras que para el plátano, el pH disminuye con el aumento de EM y la acidez titulable aumenta, producto de la degradación de almidón en azúcares reductores o su conversión en ácido pirúvico. 
Un aumento en el estado de madurez en el mango, maracuyá amarilla, papaya Hawaiana y plátano, produce un incremento en el índice de madurez, debido al aumento de los sólidos solubles y disminución de la acidez titulable.

Tabla 3: Propiedades fisicoquímicas para diferentes estados de madurez (EM) de diversas frutas tropicales (*Letras diferentes indican diferencias significativas en comparación de medias para EM).

\begin{tabular}{|c|c|c|c|c|c|}
\hline \multirow[b]{2}{*}{ Fruta } & \multirow[b]{2}{*}{ EM } & \multicolumn{4}{|c|}{ Propiedad fisicoquímica } \\
\hline & & $\mathrm{pH}$ & $\begin{array}{c}\text { Acidez titulable } \\
\mathrm{g} / 100 \mathrm{ml}\end{array}$ & $\begin{array}{c}\text { Sólidos solubles, } \\
{ }^{\circ B r i x}\end{array}$ & Índice de madurez \\
\hline \multirow{6}{*}{$\begin{array}{l}\text { Mango } \\
\text { variedad } \\
\text { puerco }\end{array}$} & 1 & $3,42 \pm 0,04^{\mathrm{a}}$ & $0,75 \pm 0,11^{\mathrm{a}}$ & $3,34 \pm 0,54^{\mathrm{a}}$ & $4,48 \pm 0,04^{a}$ \\
\hline & 2 & $3,60 \pm 0,02^{b}$ & $0,62 \pm 0,01^{\text {ab }}$ & $5,38 \pm 0,08^{b}$ & $8,59 \pm 0,01^{b}$ \\
\hline & 3 & $3,68 \pm 0,03^{c}$ & $0,60 \pm 0,03^{b}$ & $14,25 \pm 0,61^{\mathrm{c}}$ & $23,62 \pm 0,03^{\mathrm{c}}$ \\
\hline & 4 & $3,83 \pm 0,01^{d}$ & $0,45 \pm 0,02^{\mathrm{C}}$ & $15,16 \pm 0,67^{\mathrm{c}}$ & $33,71 \pm 0,01^{d}$ \\
\hline & 5 & $3,89 \pm 0,04^{d}$ & $0,42 \pm 0,03^{\mathrm{cd}}$ & $18,16 \pm 1,14^{c}$ & $43,71 \pm 0,04^{\mathrm{e}}$ \\
\hline & 6 & $3,97 \pm 0,02^{\mathrm{e}}$ & $0,30 \pm 0,01^{d}$ & $15,91 \pm 0,53^{\mathrm{d}}$ & $53,74 \pm 0,02^{\dagger}$ \\
\hline \multirow{6}{*}{$\begin{array}{l}\text { Mango } \\
\text { variedad } \\
\text { corazón }\end{array}$} & 1 & $3,51 \pm 0,04^{a}$ & $0,76 \pm 0,13^{\mathrm{a}}$ & $3,43 \pm 0,57^{a}$ & $4,54 \pm 0,03^{a}$ \\
\hline & 2 & $3,57 \pm 0,02^{\mathrm{ab}}$ & $0,65 \pm 0,02^{\mathrm{ab}}$ & $5,53 \pm 0,15^{a}$ & $8,53 \pm 0,01^{b}$ \\
\hline & 3 & $3,66 \pm 0,03^{b}$ & $0,58 \pm 0,07^{\text {bcd }}$ & $13,68 \pm 1,75^{b}$ & $23,56 \pm 0,03^{\mathrm{c}}$ \\
\hline & 4 & $3,79 \pm 0,03^{c}$ & $0,48 \pm 0,03^{\mathrm{bc}}$ & $16,28 \pm 0,88^{\mathrm{c}}$ & $33,64 \pm 0,02^{d}$ \\
\hline & 5 & $3,86 \pm 0,04^{c}$ & $0,43 \pm 0,02^{\mathrm{cd}}$ & $18,88 \pm 0,87^{\mathrm{d}}$ & $43,64 \pm 0,03^{\mathrm{e}}$ \\
\hline & 6 & $4,04 \pm 0,06^{d}$ & $0,37 \pm 0,01^{d}$ & $19,90 \pm 0,52^{\mathrm{d}}$ & $53,79 \pm 0,07^{\dagger}$ \\
\hline \multirow{6}{*}{$\begin{array}{c}\text { Maracuyá } \\
\text { variedad } \\
\text { amarilla }\end{array}$} & 1 & $2,77 \pm 0,02^{\mathrm{a}}$ & $6,29 \pm 0,37^{a}$ & $5,11 \pm 0,77^{\mathrm{a}}$ & $0,81 \pm 0,08^{a}$ \\
\hline & 2 & $2,86 \pm 0,04^{\mathrm{ab}}$ & $6,31 \pm 0,15^{\mathrm{a}}$ & $7,22 \pm 0,84^{\text {ab }}$ & $1,14 \pm 0,11^{\mathrm{ab}}$ \\
\hline & 3 & $2,95 \pm 0,05^{b}$ & $6,01 \pm 0,07^{\mathrm{ab}}$ & $9,11 \pm 1,02^{\mathrm{bc}}$ & $1,52 \pm 0,17^{\mathrm{bc}}$ \\
\hline & 4 & $3,11 \pm 0,03^{c}$ & $5,64 \pm 0,07^{\mathrm{bc}}$ & $10,44 \pm 1,02^{c}$ & $1,85 \pm 0,16^{\mathrm{c}}$ \\
\hline & 5 & $3,16 c \pm 0,01^{\mathrm{cd}}$ & $5,42 \pm 0,19^{c}$ & $15,33 \pm 0,67^{\mathrm{d}}$ & $2,83 \pm 0,11^{\mathrm{d}}$ \\
\hline & 6 & $3,26 \pm 0,07^{d}$ & $4,79 \pm 0,21^{d}$ & $15,56 \pm 1,02^{\mathrm{e}}$ & $3,25 \pm 0,33^{d}$ \\
\hline \multirow{7}{*}{$\begin{array}{l}\text { Papaya } \\
\text { Hawaiana }\end{array}$} & 1 & $5,65 \pm 0,05^{\mathrm{a}}$ & $0,15 \pm 0,00^{\mathrm{a}}$ & $6,64 \pm 0,91^{\mathrm{a}}$ & $44,29 \pm 6,08^{\mathrm{a}}$ \\
\hline & 2 & $5,77 \pm 0,02^{b}$ & $0,14 \pm 0,01^{\text {ab }}$ & $6,82 \pm 0,17^{a}$ & $50,02 \pm 3,35^{\mathrm{ab}}$ \\
\hline & 3 & $5,80 \pm 0,01^{b c}$ & $0,12 \pm 0,01^{b c}$ & $7,60 \pm 0,35^{a}$ & $61,77 \pm 5,16^{\mathrm{ab}}$ \\
\hline & 4 & $5,82 \pm 0,01^{b c}$ & $0,11 \pm 0,01^{\mathrm{cd}}$ & $8,18 \pm 0,14^{\mathrm{ab}}$ & $72,77 \pm 9,13^{\mathrm{bc}}$ \\
\hline & 5 & $5,85 \pm 0,01^{\mathrm{cd}}$ & $0,10 \pm 0,00^{\mathrm{de}}$ & $9,33 \pm 0,37^{\mathrm{bc}}$ & $93,33 \pm 3,74^{\text {cd }}$ \\
\hline & 6 & $5,90 \pm 0,02^{d}$ & $0,09 \pm 0,01^{\mathrm{e}}$ & $10,20 \pm 0,41^{\text {cd }}$ & $109,66 \pm 9,71^{d}$ \\
\hline & 7 & $5,97 \pm 0,03^{\mathrm{e}}$ & $0,09 \pm 0,01^{\mathrm{e}}$ & $11,56 \pm 1,02^{d}$ & $139,52 \pm 19,99^{\mathrm{e}}$ \\
\hline \multirow{7}{*}{ Plátano } & 1 & $5,62 \pm 0,11^{\mathrm{a}}$ & $0,13 \pm 0,02^{\mathrm{a}}$ & $1,11 \pm 0,38^{\mathrm{a}}$ & $8,61 \pm 2,21^{\mathrm{a}}$ \\
\hline & 2 & $5,45 \pm 0,12^{\mathrm{ab}}$ & $0,17 \pm 0,01^{b}$ & $6,00 \pm 0,67^{b}$ & $34,57 \pm 2,16^{b}$ \\
\hline & 3 & $5,23 \pm 0,08^{\mathrm{bc}}$ & $0,19 \pm 0,01^{b c}$ & $11,56 \pm 0,77^{\mathrm{c}}$ & $59,76 \pm 0,42^{c}$ \\
\hline & 4 & $5,05 \pm 0,09^{\text {cd }}$ & $0,22 \pm 0,01^{c d}$ & $14,22 \pm 0,77^{d}$ & $63,68 \pm 0,17^{d}$ \\
\hline & 5 & $4,93 \pm 0,03^{d}$ & $0,25 \pm 0,00^{\mathrm{de}}$ & $16,00 \pm 0,00^{d}$ & $64,00 \pm 0,00^{d}$ \\
\hline & 6 & $4,46 \pm 0,04^{\mathrm{e}}$ & $0,28 \pm 0,01^{\mathrm{e}}$ & $18,22 \pm 0,77^{\mathrm{e}}$ & $64,32 \pm 0,11^{\mathrm{d}}$ \\
\hline & 7 & $4,29 \pm 0,02^{\mathrm{e}}$ & $0,28 \pm 0,02^{\dagger}$ & $20,67 \pm 1,34^{\dagger}$ & $64,58 \pm 0,14^{\mathrm{d}}$ \\
\hline
\end{tabular}

\section{REFERENCIAS}

Abdullah, M., L. Guan, K. Lim y A.Karim, The applications of computer vision system and tomographic radar imaging for assessing physical properties of food. Journal of Food Engineering, 61 (1), 125-135 (2001).

Adobe Systems inc., Adobe Photoshop CS5® versión 12. www.adobe.com, Acceso: 4 de Abril (2012).

A.O.A.C. Oficial method of analysis.Association of Official Analiytical Chemistry. $16^{\text {th }}$ edición, Ed.By Hoorwitz, N., P. Chialo, y H. Reynold, Washington, USA (1990).

Arrieta, A., U. Baquero, y J. Barrera, Caracterización fisicoquímica del proceso de maduración del plátano "Papocho" (Musa ABB Simmonds), Agronomía Colombiana, 24(1), 48-53(2006).

Azcón-Bieto, J y M. Talón,Fundamentos de fisiología vegetal, 2ªedición,173-474. Interamericana-McGrawHill, Nueva York, USA (2008). 
Barrera, J., G. Arrazola y D.Cayón, Caracterización fisicoquímica y fisiológica del proceso de maduración de plátano Hartón (musa AAB Simmonds) en dos sistemas de producción, Acta Agronómica, 59 (1), $20-29$ (2010).

Bruhn, C.M.,Aspectos de calidad y seguridad alimentaria de interés para el consumidor. In Tecnología Poscosecha de Productos Hortofrutícolas. $3^{\mathrm{a}}$ edición. Kader, A. (Ed.), 37-44. University of California, Division of Agriculture and Natural Resources, Oakland, USA (2007).

Casierra, F yO. Aguilar,Calidad en frutos de tomate (Solanumlycopersicum L.) cosechados en diferentes estados de madurez, Agronomía Colombiana, 26(2), 300-307 (2008).

Chang-Yuen, K. y M.V. Sáenz,Efecto del 1-metil-ciclopropeno (1-mcp) en la maduración de banano, Agronomía Costarricense, 29 (3), 211-220 (2005).

Dadzie, B. y J. Orchard, Evaluación rutinaria postcosecha de híbridos de bananos y plátanos: criterios y métodos. Guías técnicas INIBAP 2. Instituto Internacional de Recursos Fitogenéticos 2, 76 (1997).

FAO.,Situación actual y perspectivas a plazo medio para las frutas tropicales. Organización de las Naciones Unidas para la Agricultura y la Alimentación. http://www.fao.org/es/esc/common/ecg/218/es/Sit_web_s.pdf. Acceso: 9 de Septiembre (2012).

Fraser, P.D., M.R. Truesdale, C.R. Bird, W. Schuch y P.M. Bramley,Carotenoid biosynthesis during tomato fruit development, Plant Physiology, 105 (1), 405-413 (1994).

León, K., D. Mery, F.Pedreschi y J. León, Color measurement in $L^{\star} a^{\star} b^{\star}$ units from RGB digital images, Food Research International, 39 (10), 1084-1091 (2006).

Mendoza, F., P. Dejmek yL. Aguilera, Calibrated color measurements of agricultural foods using image analysis, Postharvest Biology and Technology, 41 (3), 285-295 (2006).

Menéndez, O.,y otros 5 autores,Cambios en la actividad de $\alpha$-amilasa, pectinmetilesterasa $y$ poligalacturonasa durante la maduración del maracuyá amarillo (Passiflora edulis var. Flavicarpadegener), Interciencia, 31(10), 728-733 (2006).

Moccia, S., A. Chiesa, A. Oberti y P.A. Tittonell,Yield and quality of sequentially grown cherry tomato and lettuce under long-term conventional, low-input and organic soil management systems, European Journal of Horticultural Science, 71(4), 183-191 (2006).

Pedreschi, F.,D. Mery, F. Mendoza y J. Aguilera,Classification of potato chips using pattern recognition. Journal of FoodScience, 69 (6), 264-269 (2004).

Peña, M., Tratamiento cuarentenario contra la mosca mediterráneo (Ceratitiscapitata) en mangos variedad Haden (Manguífera índica) con irradiación gamma (Co-60). Magíster en Ciencia de los Alimentos, Universidad Nacional Mayor de San Marcos, Lima (2008).

Santamaría, F., y otros 5 autores, Postharvest ripening and maturity indices for maradol papaya, Interciencia, 34 (8), 583- 588 (2009).

Sañudo, A., y otros 5 autores, Control de la maduración en frutos de papaya (carica papaya l.) Con 1metilciclopropeno y ácido 2-cloroetil fosfónico,RevistaFitotecnia Mexicana, 31 (2), 141-147 (2008).

Segnini, S., P. Dejmek yR. Öste, A low cost video technique for color measurement of potato chips,LWT Food Science and Technology, 32 (4), 216-222 (1999).

Torres, R., E. Montes, O. Pérez y R.D. Andrade,Influencia del estado de madurez sobre las propiedades viscoelásticasde frutas tropicales (mango, papaya y plátano), InformaciónTecnológica, 23(5),115-124 (2012)

Yam, K y S. Papadakis,A simple digital imaging method for measuring and analyzing color of food surfaces, Journal of FoodEngineering 61 (1), 137-142 (2004) 